OPEN ACCESS

Edited by:

Chirstian Wöber,

Medical University of Vienna, Austria

Reviewed by:

Oliver Summ,

University of Oldenburg, Germany

Wouter Schievink,

Cedars Sinai Medical Center,

United States

*Correspondence:

Andreas Raabe

andreas.raabe@insel.ch

these authors have contributed equally to this work

Specialty section:

This article was submitted to Headache and Neurogenic Pain,

a section of the journal

Frontiers in Neurology

Received: 17 August 2021 Accepted: 11 October 2021 Published: 01 November 2021

Citation:

Goldberg J, Häni L, Jesse CM Zubak I, Piechowiak El, Gralla J, Dobrocky T, Beck J and Raabe A

(2021) Spontaneous Intracranial

Hypotension Without CSF Leakage-Concept of a Pathological

Cranial to Spinal Fluid Shift.

Front. Neurol. 12:760081.

doi: 10.3389/fneur.2021.760081

\section{Spontaneous Intracranial Hypotension Without CSF Leakage-Concept of a Pathological Cranial to Spinal Fluid Shift}

\author{
Johannes Goldberg ${ }^{1 \dagger}$, Levin Häni ${ }^{1+}$, Christopher Marvin Jesse ${ }^{1}$, Irena Zubak ${ }^{1}$, \\ Eike I. Piechowiak ${ }^{2}$, Jan Gralla ${ }^{2}$, Tomas Dobrocky ${ }^{2}$, Jürgen Beck ${ }^{3}$ and Andreas Raabe ${ }^{1 *}$ \\ ${ }^{1}$ Department of Neurosurgery, Inselspital, Bern University Hospital, University of Bern, Bern, Switzerland, ${ }^{2}$ University Institute \\ of Diagnostic and Interventional Neuroradiology, Inselspital, Bern University Hospital, University of Bern, Bern, Switzerland, \\ ${ }^{3}$ Department of Neurosurgery, Freiburg University Hospital, Freiburg, Germany
}

Objective: Spontaneous intracranial hypotension $(\mathrm{SIH})$ is typically caused by CSF leakage from a spinal dural tear, a meningeal diverticulum, or a CSF venous fistula. However, some patients present with classic orthostatic symptoms and typical intracranial imaging findings without evidence of CSF leakage despite repeated diagnostic work-up. This article aims to elaborate a hypothesis that would explain a pathologically increased orthostatic shift of CSF from the cranial to the spinal compartment in the absence of a CSF leak.

Medical Hypothesis: The symptoms of SIH are caused by a decrease in intracranial CSF volume, intracranial hypotension, and downward displacement of intracranial structures. A combination of pathologically increased spinal compliance, decreased intracranial CSF volume, low CSF outflow resistance, and decreased venous pressure might result in a pathological orthostatic cranial-to-spinal CSF shift. Thus, in rare cases, intracranial hypotension may occur in the absence of CSF leakage from the dural sac.

Conclusion: We propose a pathophysiological concept for the subgroup of $\mathrm{SIH}$ patients with typical cranial imaging findings and no evidence of CSF leakage. In these patients, reducing the compliance or the volume of the spinal compartment seems to be the appropriate therapeutic strategy.

Keywords: spontaneous intracranial hypotension, compliance, CSF physiology and anatomy, CSF leak or fistula, orthostatic headaches

\section{INTRODUCTION}

Orthostatic headache is the hallmark of headache attributed to spontaneous intracranial hypotension (SIH) as defined by the third edition of the international classification of headache disorders (ICHD-3) category 7.2.3 (1). The effects of the disease can be devastating and cause disability. Leakage of CSF from a defect in the spinal dura mater is responsible for a pathological decrease of CSF volume (2-4). Intracranial CSF volume depletion when in an upright position leads to a pathological decrease of intracranial pressure (ICP), brain sagging, venous distension (5), and related symptoms such as orthostatic headache and cranial nerve deficits $(2,6)$. In patients with persistent SIH symptoms, invasive diagnostics such as dynamic myelography and CT-myelography 


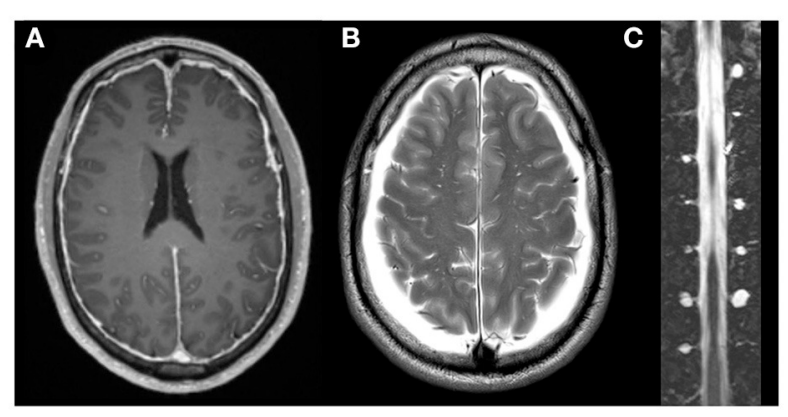

FIGURE 1 | Imaging of a patient with SIH symptoms without evidence of CSF leakage or CSF venous fistula. A 68-year-old male patient exhibiting classic $\mathrm{SIH}$ symptoms with typical cranial imaging findings, such as dural enhancement (A) and bilateral subdural hygroma (B) and no evidence of CSF leakage or CSF venous fistula despite repeated diagnostic work-up. Spinal MRI revealed a large number of prominent meningeal diverticula (C).

are recommended to search for spinal CSF leaks that are amenable to surgical treatment like ventral dural tears, ruptured meningeal diverticula, or a direct CSF to venous fistula (4, 711). However, in some patients with intractable SIH symptoms and typical cranial imaging findings (12), no evidence of spinal CSF leakage can be found despite repeated diagnostic work-up (Figure 1). In our experience, this subgroup accounts for up to $10-15 \%$ of patients, which is in line with the observations of Schievink et al. (13). Apart from symptomatic treatment, appropriate further management of these patients is unclear.

Orthostatic headache without CSF leakage due to a pathologically increased spinal compliance was first discussed by Hunderfund and Mokri (14). However, it is not yet fully understood and is rarely considered in clinical practice. In this paper, we sought to corroborate the hypothesis of a pathological cranial-to-spinal CSF shift without CSF leakage, to consider the factors that may contribute, and to discuss the diagnostic and therapeutic options.

Figures 2-4 are displaying the physiological and pathophysiological concepts of CSF dynamics relevant for $\mathrm{SIH}$ that are going to be elaborated in detail in the following.

\section{PHYSIOLOGY OF CRANIOSPINAL CSF DYNAMICS RELEVANT FOR SIH}

\section{Physiological Intracranial and Intraspinal Pressure Dynamics During Positional Changes}

In a person in a recumbent position, the CSF pressure is equal along the craniospinal axis. Thus, the opening pressure during lumbar puncture in a recumbent position corresponds to the ICP. When moving to an upright position, the pressure redistributes along the hydrostatic column from negative values in the cranial compartment to positive values in the spinal compartment (15). The zero pressure point, i.e., the transition point from negative

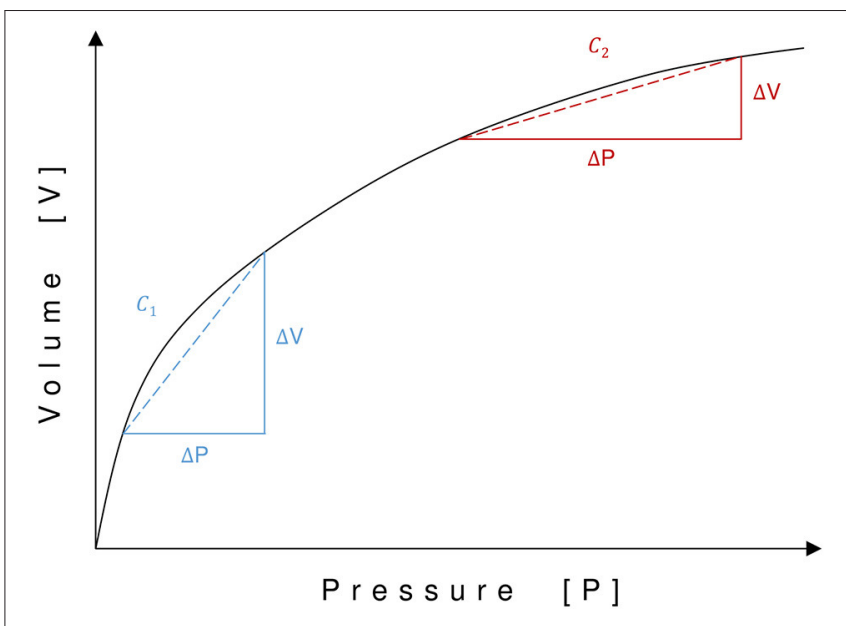

FIGURE 2 | Pressure-volume curve of the craniospinal compartment. Pressure $(P)$ is displayed on the $x$-axis and volume $(V)$ on the $y$-axis. Compliance $(C)$ is represented by the slope of the pressure-volume curve. Due to its exponential function, compliance varies with different pressure-volume status. A shift to the left on the pressure-volume curve leads to increased compliance (C1), whereas a shift to the right leads to decreased compliance $(C 2)$.

to positive pressure values, is located in the upper cervical spine (15). The point where the pressure in the upright position is identical to the pressure in the recumbent position is called the hydrostatic indifferent point (HIP). It is physiologically situated between C7 and T5 (15). The physiological ICP in the upright position is slightly negative (16-18).

\section{Craniospinal Compliance}

Craniospinal compliance $(C)$ is a measure of distensibility. It describes the ratio between changes in volume and pressure ( $C=\frac{\Delta V}{\Delta P}$ ) and corresponds to the slope of the pressure-volume curve. Since the relationship between pressure and volume in the CSF space is exponential, compliance increases with a lower filling pressure or a lower CSF volume and vice versa $(19,20)$. Thus, in a state of high baseline pressure, a change in volume results in a relatively larger change of pressure than in a state of low baseline pressure (Figure 2).

It is essential to understand the normal movements of CSF between the cranial and the spinal compartment caused by postural changes. The craniospinal CSF system of adults accommodates $\sim 150(125-200) \mathrm{ml}$ (21). Roughly two-thirds of the CSF volume is located in the spinal and one-third in the cranial compartment. When moving from a recumbent to an upright position, the pressure above the HIP decreases and the pressure below the HIP increases. Because of the compliance of the thecal sac, the spinal compartment accommodates some additional CSF volume, causing a CSF shift from the cranial to the spinal compartment, and a decrease in ICP. Provided that the spinal compliance is physiological, the cranial-to-spinal CSF shift is limited to a few milliliters of CSF and the decrease in ICP remains within a physiological range (22), which is not associated with orthostatic headache (Figure 3A). 


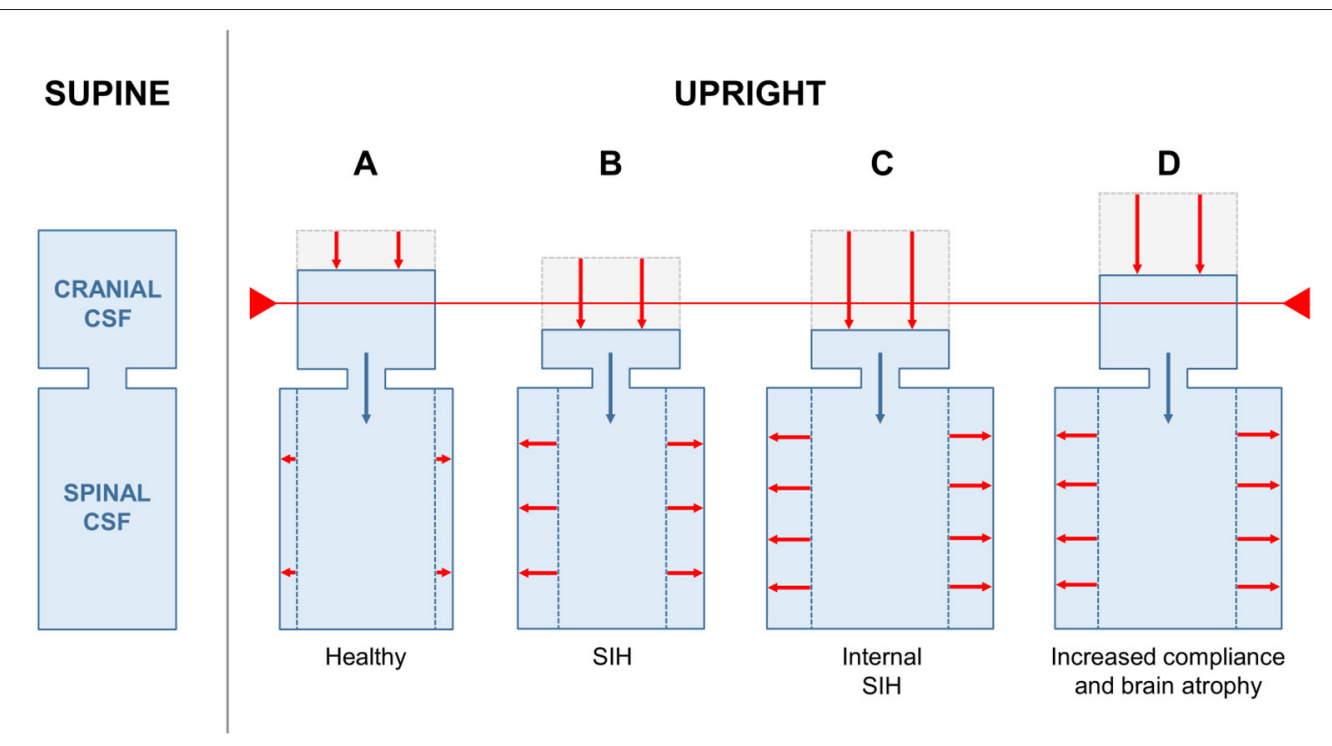

FIGURE 3 | Hypothetical CSF volume shifts in different constellations of SIH and healthy individuals. Schematic illustration of craniospinal CSF distribution in the supine (left) and upright position (right; A-D). The red line indicates the threshold for development of orthostatic symptoms. In a healthy individual only a small proportion of the cranial CSF volume is shifted to the spinal compartment after a positional change (A). CSF leakage in patients with classical SIH leads to CSF hypovolemia and a shift to the left on the pressure-volume curve with increased compliance. Thus, a larger volume (absolute and proportional) is shifted to the spinal compartment (B). In a state of increased spinal compliance, a larger absolute volume is shifted to the spinal compartment (C,D). In patients with normal or low intracranial CSF volume, the proportion of CSF shifted is large and causes internal SIH (C). In patients with high intracranial CSF volume (e.g., because of brain atrophy) the proportionally smaller CSF shift is compensated and no symptoms occur (D).

\section{PATHOPHYSIOLOGY OF CSF DYNAMICS IN SIH}

\section{Pathophysiology of Intracranial Pressure Changes in SIH Patients With Proven CSF Leakage}

In patients with CSF leakage, the total craniospinal CSF volume is reduced, and the pressure decreased (23). Therefore, the state of the craniospinal compartment shifts to the left on the pressure-volume curve with increased compliance (Figure 2). When a patient with CSF leakage moves from a recumbent to an upright position, because of its increased compliance, the spinal compartment can accommodate more CSF. Thus, a larger proportion of the already diminished intracranial CSF volume is now shifted into the spinal compartment, and a pathologically low ICP, brain sagging, and orthostatic headache and other symptoms (Figure 3B) can occur. In patients who experience orthostatic headache after lumbar puncture or due to overdraining CSF shunts, such a pathologically low ICP can decrease to $-35 \mathrm{cmH}_{2} \mathrm{O}$ in an upright position $(16,17,24)$.

Due to the equal distribution of pressure and fluid when in a horizontal position, the reduced CSF volume and the increased compliance have only a minor effect on ICP in the recumbent position. Measurements of opening lumbar pressure of SIH patients in the recumbent position demonstrate lower (i.e., $<6$ $\mathrm{cmH}_{2} \mathrm{O}$ ) or most often normal but not negative pressure. Thus, in the recumbent position, the intracranial pressure is positive, even in patients with CSF loss, which prevents brain sagging and explains the immediate relief experienced when the patient is lying down.

\section{THE HYPOTHESIS OF INTERNAL SIH-FACTORS CONTRIBUTING TO INTRACRANIAL HYPOTENSION WITHOUT CSF LEAKAGE}

We hypothesize that a pathologically increased compliance of the spinal compartment with increased orthostatic CSF shift from the cranial to the spinal compartment can cause intracranial hypotension and brain sagging in the absence of CSF leakage. Together with predisposing factors, such as low total and low intracranial CSF volume, decreased CSF outflow resistance, and decreased intracranial venous pressure, orthostatic symptoms can occur. We believe that this concept applies to patients exhibiting classic SIH symptoms with typical intracranial imaging findings, but no evidence of CSF leakage despite repeated diagnostic work-up. We will refer to this as internal SIH in the following sections.

\section{Increased Compliance of the Spinal Compartment}

The volume of CSF shifting from the cranial toward the spinal compartment during a postural change depends mostly on the compliance and thus on the volume reserve of the spinal compartment, the latter being the main contributor 


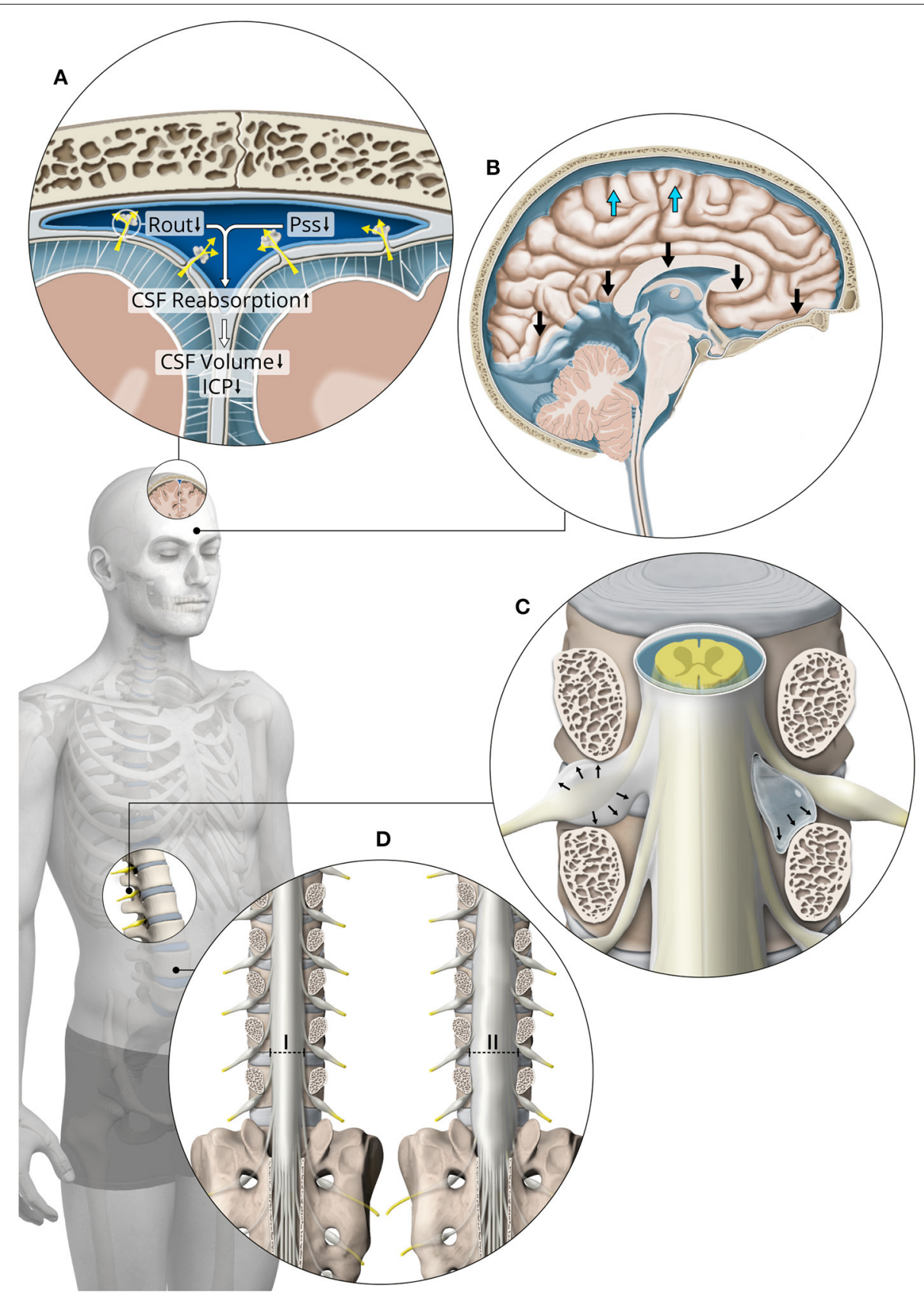

FIGURE 4 | Overview of factors contributing to internal SIH. Predisposing factors are (A) low CSF outflow resistance (Rout), low intracranial venous pressure (Pss) and (B) low intracranial CSF volume. The main pathophysiological factor is increased spinal compliance due to (C) meningeal diverticula, dural tears with prolapsing arachnoid around meningeal diverticula or (D) increased distensibility of the dura mater ( $I=$ normal distensibility, $I I=$ increased distensibility). A combination of these factors can lead to a pathological cranial-to-spinal CSF shift causing brain sagging (B) and orthostatic symptoms without CSF leakage.

to the total craniospinal compliance (25). In an a priori pathological increase in spinal compliance, the volume of CSF shifting from the cranial to the spinal compartment may be large enough to cause $\mathrm{SIH}$, brain sagging, and symptoms, without CSF leakage to the extradural space (Figure 3C). We consider this the major pathophysiological factor for internal SIH.
Potential explanations for pathologically increased compliance are (1) a large number of meningeal diverticula (Figure 1C), (2) dural tears with prolapsing arachnoid around meningeal diverticula or elsewhere (Figure 4C), and (3) increased distensibility of the dura mater (Figure 4D) $(4,26,27)$. According to Schievink, meningeal diverticula of $8 \mathrm{~mm}$ or more are accountable for $42 \%$ of cases of SIH. However, 
an associated extradural CSF collection was found in only $22 \%$ of these cases (13). A tear in the dura mater may lead to a prolapse of the arachnoid, which is frequently found intra-operatively when exploring meningeal diverticula. The prolapsing arachnoid could potentially increase the compliance of the spinal CSF compartment. To our knowledge, imaging of those diverticula has only been done in the supine position, and it is not known whether ballooning of diverticula when in the upright position might increase the compliance of the spinal compartment. Additionally, Kranz et al. found significantly more meningeal diverticula in SIH patients without evidence of CSF leakage than in SIH patients with a proven CSF leak (28). Therefore, we hypothesize that meningeal diverticula can result in increased compliance of the spinal compartment, leading to SIH symptoms, despite the absence of CSF leakage.

\section{Low Total CSF Volume}

In the case of an overall reduction in CSF volume, the state of the craniospinal compartment shifts to the left on the pressurevolume curve, leading to increased compliance (Figure 2). This might allow for a pathologically increased shift of CSF volume to the spinal compartment when moving to an upright position.

\section{Low Intracranial CSF Volume}

The intracranial CSF volume varies according to the size of the ventricles and the volume of the subarachnoid space. It is agedependent but also related to a variety of factors causing brain atrophy. If the brain is atrophic, the size of the intracranial CSF compartment is increased. For example, provided that the same amount of volume in milliliters shifts to the spinal compartment during positional changes, in patients with higher intracranial CSF volume the proportion of CSF shifted is lower and more CSF remains in the intracranial space (Figure 3D). Conversely, in patients with lower intracranial CSF volume, the proportion of CSF shifted is higher and less CSF remains in the intracranial space (Figure 3C). A smaller intracranial CSF volume predisposes an individual to a reduced buoyancy of the brain, brain sagging and increased tension on pain sensitive structures like meninges and dural sinuses (Figure 4B). The contribution of smaller intracranial CSF volume is supported by the age distribution of SIH patients who are seldom elderly.

\section{CSF Outflow Resistance}

ICP in the recumbent position is a function of CSF formation rate $\left(I_{f}\right)$, CSF outflow resistance $\left(R_{\text {out }}\right)$, and sagittal sinus pressure $\left(P_{\mathrm{ss}}\right)$, as defined in Davson's equation: (17).

$$
I C P=I_{f} * R_{\text {out }}+P_{s s}
$$

The outflow of CSF is directly proportional to the pressure difference between ICP and $P_{\mathrm{ss}}$. The proportionality constant is $R_{\text {out }}(29,30)$. $R_{\text {out }}$ can be determined by continuous lumbar infusion testing (31). Decreased $R_{\text {out }}$ leads to lower CSF volume and pressure due to increased CSF reabsorption (Figure 4A). Therefore, the state of the craniospinal compartment shifts to the left on the pressure-volume curve with increased compliance.

A decrease of $R_{\text {out }}$ might occur in the form of a spinal CSF venous fistula. Although rare it is a recognized cause for the development of SIH $(13,32,33)$. Another hypothesis of unknown clinical relevance is a pathologically decreased $R_{\text {out }}$ caused by high resorption capacity of the spinal meningeal roots. Although they play a role in CSF reabsorption, their normally constant and unchanged appearance does not sufficiently explain the sudden onset of SIH symptoms. However, multiple meningeal diverticula around spinal nerve roots might form throughout life and are a potential cause of an acquired increased CSF reabsorption capacity and sudden onset of SIH symptoms. However, this is speculative and needs to be proven.

\section{Decreased Venous Pressure}

If $R_{\text {out }}$ remains constant, according to the Davson's equation a decrease in intracranial or intraspinal venous pressure will lead to increased CSF reabsorption, reduced CSF volume, lower ICP, and a shift toward the left on the pressure-volume curve with increased compliance (Figure 4A). Moreover, a reduction of spinal epidural venous filling allows the thecal sac to expand more easily in case of volume load. Kumar and colleagues reported a case of intracranial hypotension associated with inferior vena cava stenosis (34). Low intracaval pressure has been proposed to cause increased CSF reabsorption from the spinal compartment to the inferior vena cava via the epidural venous plexus $(34,35)$. Symptoms and imaging findings resolved after stenting of the stenotic segment.

\section{DISCUSSION}

We describe the hypotheses of a pathological, orthostatic cranial-to-spinal CSF shift resulting in the clinical picture of symptomatic intracranial hypotension without evidence of CSF leakage, which we refer to as internal SIH. Based upon the interaction of pathologically increased compliance of the spinal compartment with predisposing factors such as decreased total and intracranial CSF volume, decreased CSF outflow resistance, and decreased venous pressure, intracranial hypotension and brain sagging may develop. This hypothesis is important because the contributing pathophysiological factors could ultimately serve as diagnostic and therapeutic targets.

\section{Criteria Qualifying for the Suspicion of Internal SIH}

We propose the following combination of criteria for the suspicion of internal SIH:

1. Classic SIH symptoms

2. Typical cranial imaging findings (12)

3. Absence of evidence of CSF leakage or CSF venous fistula despite repeated spinal imaging after intrathecal contrast application (MR-myelography, CT-myelography, dynamic digital subtraction myelography) (4)

4. Clear benefit from unspecific epidural blood patch (EBP), upon repeated applications.

These criteria do not provide direct proof and thus allow only the suspicion of internal SIH. Several problems need to be considered when trying to establish this diagnosis: First, because of the limited sensitivity of MR-myelography, CT-myelography, 
dynamic CT-myelography, and digital subtraction myelography, it is not possible to exclude an existing, but undetected small dural leak or CSF venous fistula. Thus, repeated and targeted work-up is required before considering the diagnosis of internal SIH.

Second, the above-mentioned factors-spinal compliance, total CSF volume, intracranial CSF volume, CSF outflow resistance, and low venous pressure-are relevant to the hypothesis of internal SIH. However, routine testing of these factors is not yet accepted in clinical practice. Furthermore, no specific test with diagnostic thresholds exists for these parameters. For instance, even the most promising theoretical parameter, $\mathrm{R}_{\text {out }}$, shows considerable variations and timedependent behavior (31).

Direct proof could be obtained from measurement of pathologically increased compliance by using the opening pressure and the volume-pressure response, and by ICP measurement while moving into an upright position. Indeed, some patients with severe intractable symptoms would qualify for invasive and dynamic ICP measurement with an implanted telemetric ICP probe. However, the insertion of these devices exposes the patient to a relevant risk of neurological deficits $(0.4 \%)$, seizures $(4.5 \%)$, and infections (1.6\%) (36). Hence, physicians have been reluctant to use them as a diagnostic tool in headache attributed to spontaneous intracranial hypotension.

From a pathophysiological point of view, we also advocate measurement of $\mathrm{R}_{\text {out }}$ by lumbar infusion testing, and CSF volumetry of cranial and spinal spaces in the diagnostic work-up of these patients.

\section{Therapeutic Options}

Of all the contributing factors described above, addressing the pathologically increased spinal compliance and thus the spinal volume reservoir is most feasible. A simple and well-tolerated method to achieve this is $\operatorname{EBP}(37,38)$. The temporary relief of $\mathrm{SIH}$ symptoms after unspecific EBP is in line with the hypothesis of internal SIH. Increasing the volume of the epidural space with an EBP leads to compression of the dural sac, reduction of the volume capacity and decreased compliance of the spinal CSF compartment. Thus, the volume of CSF that shifts from the cranial to the spinal compartment during a postural change is smaller and more CSF remains in the intracranial space. The temporary nature of the relief is most likely explained by the absorption of blood from the epidural space over time and the restoration of the increased compliance. For patients

\section{REFERENCES}

1. Headache Classification Committee of the International Headache Society (IHS). The International classification of headache disorders, 3rd edition. Cephalalgia. (2018) 38:1-211. doi: 10.1177/0333102417738202

2. Ducros A, Biousse V. Headache arising from idiopathic changes in CSF pressure. Lancet Neurol. (2015) 14:65568. doi: 10.1016/S1474-4422(15)00015-0

3. Schievink WI. Spontaneous spinal cerebrospinal fluid leaks and intracranial hypotension. JAMA. (2006) 295:2286-96. doi: 10.1001/jama.295.19.2286 with a good but temporary clinical response, repeated EBP is an option. In patients with a sustained response to EBP, the blood might have initiated a process of scarring in the epidural space and/or the spinal dura mater, thereby diminishing the compliance of the spinal compartment, or the site of leakage could have closed spontaneously.

Another therapeutic option to reduce compliance of the spinal compartment is reduction of prominent meningeal diverticula or dural reduction surgery. The principle of the surgery is to reduce the volume capacity of the spinal CSF reservoir and thus to decrease the compliance of the spinal compartment. Consequently, a smaller volume of CSF is shifted from the cranial to the spinal compartment when assuming an upright position. Schievink et al. demonstrated a positive effect of lumbar dural reduction surgery in a patient with intractable SIH but no evidence for CSF leakage (39). This is an invasive method, however, which should be reserved as a last option for selected patients.

\section{CONCLUSION}

We propose a pathophysiological concept for the subgroup of SIH patients with typical cranial imaging findings and no evidence of CSF leakage after repeated and meticulous work-up. Pathologically increased spinal compliance as the main factor, in combination with predisposing factors such as decreased total and intracranial CSF volume, low CSF outflow resistance, and decreased venous pressure might cause a pathological orthostatic CSF shift resulting in intracranial hypotension and brain sagging even in the absence of CSF leakage. The compliance of the spinal compartment seems to be the most attractive therapeutic target.

\section{DATA AVAILABILITY STATEMENT}

The original contributions presented in the study are included in the article, further inquiries can be directed to the corresponding author/s.

\section{AUTHOR CONTRIBUTIONS}

JGo, LH, JB, and AR: design and conceptualized study and drafted manuscript. CJ, IZ, EP, JGr, and TD: revised manuscript for intellectual content. All authors contributed to the article and approved the submitted version. 
8. Schievink WI. Spontaneous spinal cerebrospinal fluid leaks. Cephalalgia. (2008) 28:1345-56. doi: 10.1111/j.1468-2982.2008.01776.x

9. Schievink WI, Reimer R, Folger WN. Surgical treatment of spontaneous intracranial hypotension associated with a spinal arachnoid diverticulum: case report. J Neurosurg. (1994) 80:736-9. doi: 10.3171/jns.1994.80.4.0736

10. Schievink WI, Morreale VM, Atkinson JL, Meyer FB, Piepgras DG, Ebersold MJ. Surgical treatment of spontaneous spinal cerebrospinal fluid leaks. $J$ Neurosurg. (1998) 88:243-6.

11. Schievink WI, Meyer FB, Atkinson JL, Mokri B. Spontaneous spinal cerebrospinal fluid leaks and intracranial hypotension. I Neurosurg. (1996) 84:598-605.

12. Dobrocky T, Grunder L, Breiding PS, Branca M, Limacher A, Mosimann PJ, et al. Assessing spinal cerebrospinal fluid leaks in spontaneous intracranial hypotension with a scoring system based on brain magnetic resonance imaging findings. JAMA Neurol. (2019) 76:580-7. doi: 10.1001/jamaneurol.2018.4921

13. Schievink WI, Maya MM, Jean-Pierre S, Nuño M, Prasad RS, Moser FG. A classification system of spontaneous spinal CSF leaks. Neurology. (2016) 87:673-9. doi: 10.1212/WNL.0000000000002986

14. Leep Hunderfund AN, Mokri B. Orthostatic headache without CSF leak. Neurology. (2008) 71:1902-6. doi: 10.1212/01.wnl.0000336655.07 617.e0

15. Magnaes B. Body position and cerebrospinal fluid pressure: Part 2: clinical studies on orthostatic pressure and the hydrostatic indifferent point. $J$ Neurosurg. (1976) 44:698-705. doi: 10.3171/jns.1976.44.6.0698

16. Chapman PH, Cosman ER, Arnold MA. The relationship between ventricular fluid pressure and body position in normal subjects and subjects with shunts: a telemetric study. Neurosurgery. (1990) 26:181-9.

17. Fox JL, McCullough DC, Green RC. Effect of cerebrospinal fluid shunts on intracranial pressure and on cerebrospinal fluid dynamics: 2. A new technique of pressure measurements: results and concepts 3. A concept of hydrocephalus. J Neurol Neurosurg Psychiatry. (1973) 36:302-12. doi: 10.1136/jnnp.36.2.302

18. Petersen LG, Petersen JCG, Andresen M, Secher NH, Juhler M. Postural influence on intracranial and cerebral perfusion pressure in ambulatory neurosurgical patients. Am J Physiol Regul Integr Comp Physiol. (2016) 310:R100-R4. doi: 10.1152/ajpregu.00302.2015

19. Miller J, Garibi J, Pickard J. A clinical study of intracranial volume pressure relationships. Br J Surg. (1973) 60:316.

20. Miller J, Pickard J. Intracranial volume/pressure studies in patients with head injury. Injury. (1974) 5:265-9.

21. Brodbelt A, Stoodley M. CSF pathways: a review. $\mathrm{Br} J$ Neurosurg. (2007) 21:510-20.

22. Magnæs B. Clinical studies of cranial and spinal compliance and the craniospinal flow of cerebrospinal fluid. Br J Neurosurg. (1989) 3:659-68.

23. Dobrocky T, Rebsamen M, Rummel C, Hani L, Mordasini P, Raabe A, et al. Monro-Kellie hypothesis: increase of ventricular CSF volume after surgical closure of a spinal dural leak in patients with spontaneous intracranial hypotension. AJNR Am J Neuroradiol. (2020) 41:2055-61. doi: 10.3174/ajnr.A6782

24. McCullough DC, Fox JL. Negative intracranial pressure hydrocephalus in adults with shunts and its relationship to the production of subdural hematoma. J Neurosurg. (1974) 40:372-5.

25. Lofgren J, Zwetnow NN. Cranial and spinal components of the cerebrospinal fluid pressure-volume curve. Acta Neurol Scand. (1973) 49:575-85.

26. Mokri B, Maher CO, Sencakova D. Spontaneous CSF leaks: underlying disorder of connective tissue. Neurology. (2002) 58:814-6. doi: 10.1212/WNL.58.5.814

27. Schievink WI, Gordon OK, Tourje J. Connective tissue disorders with spontaneous spinal cerebrospinal fluid leaks and intracranial hypotension: a prospective study. Neurosurgery. (2004) 54:6571. doi: 10.1227/01.NEU.0000097200.18478.7B
28. Kranz P, Stinnett S, Huang K, Gray L. Spinal meningeal diverticula in spontaneous intracranial hypotension: analysis of prevalence and myelographic appearance. AJNR Am J Neuroradiol. (2013) 34:12849. doi: 10.3174/ajnr.A3359

29. Czosnyka M, Batorski L, Laniewski P, Maksymowicz W, Koszewski W, Zaworski W. A computer system for the identification of the cerebrospinal compensatory model. Acta Neurochir. (1990) 105:1126. doi: 10.1007/BF01669992

30. Marmarou A, Shulman K, Lamorgese J. Compartmental analysis of compliance and outflow resistance of the cerebrospinal fluid system. $J$ Neurosurg. (1975) 43:523-34.

31. Häni L, Fung $C$, Jesse $C M$, Ulrich $C T$, Miesbach $T$, Cipriani $\mathrm{DR}$, et al. Insights into the natural history of spontaneous intracranial hypotension from infusion testing. Neurology. (2020) 95:e247-55. doi: 10.1212/WNL.0000000000009812

32. Kumar N, Diehn FE, Carr CM, Verdoorn JT, Garza I, Luetmer $\mathrm{PH}$, et al. Spinal CSF venous fistula: a treatable etiology for CSF leaks in craniospinal hypovolemia. Neurology. (2016) 86:2310-2. doi: 10.1212/WNL.0000000000002776

33. Schievink WI, Moser FG, Maya MM. CSF-venous fistula in spontaneous intracranial hypotension. Neurology. (2014) 83:472-3. doi: 10.1212/WNL.0000000000000639

34. Kumar N, Neidert NB, Diehn FE, Campeau NG, Morris JM, Bjarnason H. A novel etiology for craniospinal hypovolemia: a case of inferior vena cava obstruction. J Neurosurg. (2018) 29:452-5. doi: 10.3171/2018.2.SPINE171373

35. Franzini A, Messina G, Nazzi V, Mea E, Leone M, Chiapparini L, et al. Spontaneous intracranial hypotension syndrome: a novel speculative physiopathological hypothesis and a novel patch method in a series of 28 consecutive patients. J Neurosurg. (2010) 112:300-6. doi: 10.3171/2009.6.JNS09415

36. Antes S, Tschan CA, Heckelmann M, Breuskin D, Oertel J. Telemetric intracranial pressure monitoring with the Raumedic Neurovent P-tel. World Neurosurg. (2016) 91:133-48. doi: 10.1016/j.wneu.2016.03.096

37. Wu J-W, Hseu S-S, Fuh J-L, Lirng J-F, Wang Y-F, Chen W$\mathrm{T}$, et al. Factors predicting response to the first epidural blood patch in spontaneous intracranial hypotension. Brain. (2017) 140:344-52. doi: 10.1093/brain/aww328

38. Berroir S, Loisel B, Ducros A, Boukobza M, Tzourio C, Valade D, et al. Early epidural blood patch in spontaneous intracranial hypotension. Neurology. (2004) 63:1950-1. doi: 10.1212/01.WNL.0000144339.34733.E9

39. Schievink WI. A novel technique for treatment of intractable spontaneous intracranial hypotension: lumbar dural reduction surgery. Headache. (2009) 49:1047-51. doi: 10.1111/j.1526-4610.2009.01450.x

Conflict of Interest: The authors declare that the research was conducted in the absence of any commercial or financial relationships that could be construed as a potential conflict of interest.

Publisher's Note: All claims expressed in this article are solely those of the authors and do not necessarily represent those of their affiliated organizations, or those of the publisher, the editors and the reviewers. Any product that may be evaluated in this article, or claim that may be made by its manufacturer, is not guaranteed or endorsed by the publisher.

Copyright $\odot 2021$ Goldberg, Häni, Jesse, Zubak, Piechowiak, Gralla, Dobrocky, Beck and Raabe. This is an open-access article distributed under the terms of the Creative Commons Attribution License (CC BY). The use, distribution or reproduction in other forums is permitted, provided the original author(s) and the copyright owner(s) are credited and that the original publication in this journal is cited, in accordance with accepted academic practice. No use, distribution or reproduction is permitted which does not comply with these terms. 